\title{
Determinação da Glicemia, ou como a Química Ajuda a Melhorar a Qualidade de Vida das Pessoas com Diabetes
}

João Valente Nabais

\author{
Determination of Blood Glucose, or how \\ Chemistry Helps to Improve the Quality of \\ Life of People with Diabetes. In this paper, \\ the main methods for the determination of \\ blood glucose are briefly described. Historical, \\ current and future methods are presented \\ giving special emphasis to its importance for \\ the quality of life of people with diabetes.
}

\author{
Neste artigo são sucintamente \\ descritos os principais métodos \\ para a determinação da \\ glicemia; apresentam-se métodos \\ históricos, atuais e futuros \\ dando especial destaque à sua \\ importância na qualidade de vida \\ das pessoas com diabetes.
}

\section{O que é a diabetes}

Do processo de digestão de alimentos com hidratos de carbono é formada a g'lucose que, após ser absorvida no intestino, entra na circulação sanguínea ficando disponível para ser utilizada pelas células na produção de energia. 0 transporte da glucose para o interior das células é efetuado por um processo bioquímico onde a insulina, hormona produzida nas células beta dos ilhéus de Langerhans do pâncreas, tem um papel crucial. A diabetes é uma doença metabólica crónica que se traduz por uma elevação da concentração de g'lucose no sangue, designada de hiperǵlicemia, a qual é motivada pela incapacidade do organismo utilizar convenientemente a glucose. A ausência ou o uso ineficaz da insulina leva a que a glucose fique em excesso na circulação sanguínea sendo depois expelida pela urina, num processo designado de glicosúria [1].

É curioso referir que Paul Langerhans (18471888), autor da descoberta das células produtoras de insulina, viveu na ilha da Madeira desde 1874, local escolhido pelo clima ameno da ilha, para curar uma tuberculose pulmonar e onde acabou por falecer a 20 de julho de 1888 [1].
Numa pessoa sem diabetes, quando existe um aumento da glicemia (concentração de g'lucose no sangue), o pâncreas produz a quantidade de insulina necessária para levar a glucose ao interior das células e ao seu armazenamento no fígado na forma de glicogénio. Numa pessoa com diabetes tipo 1 a produção de insulina não existe pelo facto de as células beta terem sido destruídas pelo sistema imunológico, sendo por isso designada de doença autoimune, enquanto que no caso da diabetes tipo 2 pode existir uma produção insuficiente de insulina e/ou haver uma ineficaz utilização da insulina pelo organismo, normalmente por haver insulinorresistência. Para além destes dois tipos de diabetes, que correspondem a cerca de $90 \%$ das pessoas com esta doença, há outros tipos de diabetes, como por exemplo diabetes gestacional, diabetes autoimune latente do adulto (LADA, do inglês Latent Autoimmune Diabetes in Adults) e diabetes monogénica (MODY, do inglês Maturity Onset Diabetes of the Young). Os sintomas mais frequentes da diabetes são perda de peso, falta de energia, sede em excesso, urinar 
frequentemente, infeções recorrentes, aumento de apetite, cansaço, sono excessivo e visão embaçada ou turva. No caso da diabetes tipo 2 as pessoas podem estar assintomáticas durante anos. 0 tratamento da diabetes tipo 1 é efetuado pela administração de insulina, por injeção ou perfusão contínua, enquanto que na diabetes tipo 2 são usados preferencialmente antidiabéticos orais, podendo também ser usada insulina. Para além destes fármacos é fundamental a educação terapêutica das pessoas, o exercício físico e a alimentação equilibrada.

Os critérios de diagnóstico da diabetes são definidos pela Norma 002/2011 da Direção Geral da Saúde (DGS) com base nos valores de g'licemia obtidos em determinadas condições. Os interessados podem consultar e descarregar a norma a partir do sítio de internet da DGS [2]. No que diz respeito à prevalência da diabetes, a Federação Internacional da Diabetes estima que em 2019 viviam com diabetes 463 milhões de pessoas em todo o Mundo e 59 milhões na Europa [3]. Em Portugal, o Observatório Nacional da Diabetes estima que em 2015 a prevalência na população portuguesa com idades no intervalo 20-79 anos tenha sido de $13,3 \%[4]$.

\section{Importância da determinação da glicemia}

Um dos objetivos terapêuticos das pessoas com diabetes é terem valores de glicemia dentro da gama de valores considerados ótimos no maior intervalo de tempo possível, o designado tempo no alvo. Está demonstrado, por diversos estudos, que atingir este objetivo previne, ou retarda, o surgimento das complicações da diabetes, tais como falência renal, doenças cardiovasculares, amputações e cegueira [5]. Para além destas complicações, resultantes da má compensação metabólica da diabetes durante um período mais ou menos longo, podem também surgir as complicações agudas da doença, só possíveis de detetar pela determinação da glicemia, designadamente a hiperg'licemia e a hipog'licemia. 0 caso da hipoglicemia é de particular importância pois, se não for tratada a tempo pode levar, entre outras consequências, à disfunção cognitiva e mesmo ao coma e à morte. Valores de glicemia abaixo de $70 \mathrm{mg} / \mathrm{dL}$ requerem a ingestão de açúcar ou de uma bebida açucarada. Para saber mais, pode consultar a página de internet da Associação Protetora dos Diabéticos de Portugal (APDP) em apdp.pt. De realçar ainda que as pessoas tratadas com insulina ajustam a dose consoante diversos fatores, tais como a ǵlicemia, a alimentação, o alvo terapêutico e a sensibilidade à insulina, definida como a sensibilidade do corpo aos efeitos da insulina. Esta sensibilidade é traduzida no fator de sensibilidade que quantifica qual a diminuição da ǵlicemia por cada unidade de insulina administrada.
Por estes motivos, pode dizer-se que a medição da ǵlicemia é uma ferramenta indispensável e fundamental para a gestão da diabetes e para a qualidade de vida das pessoas com esta doença. A determinação da g'licemia é feita normalmente pelas pessoas com diabetes, ou cuidadores, com o recurso a diversas técnicas e dispositivos abordados neste artigo.

\section{Um pouco de história}

Antes de ser possível determinar a g'icemia, o diagnóstico e gestão da diabetes eram efetuados pela glicosúria, glucose excretada pela urina, por exemplo através de diversos testes químicos qualitativos que usavam as propriedades redutoras da g'lucose e a reação com sulfato de cobre(II) para produzir óxido de cobre(I), cuja cor característica indicava a presença de glucose [6]. A primeira tira-teste para a análise qualitativa de g'licosúria foi desenvolvida em 1850 [6], tendo sido desde então desenvolvidas diversas tiras-teste e fitas semiquantitativas, tal como a ilustrada na Figura 1.

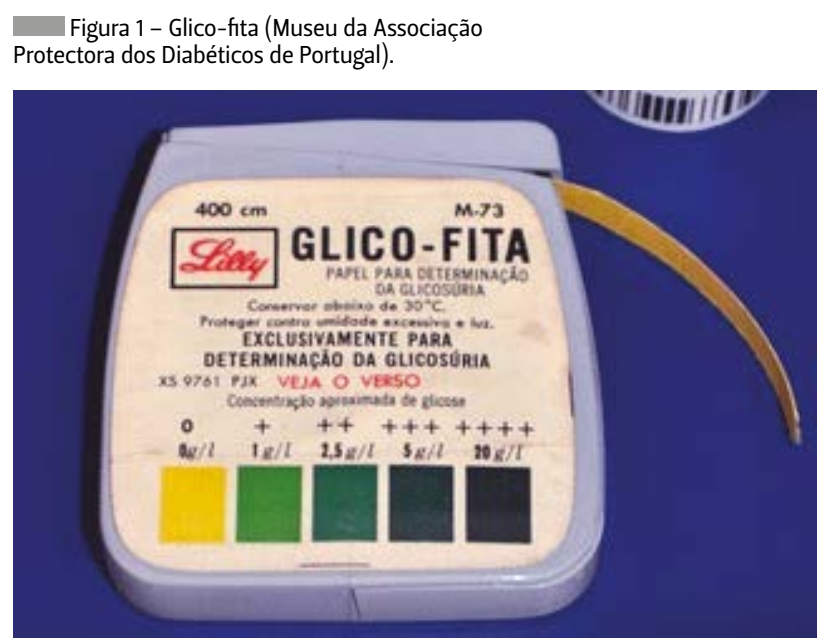

A g'licemia é determinada pelo uso de uma gota de sangue, obtida por punção capilar de um dos dedos da mão, a qual é colocada numa tira-teste. 0 sangue vai reagir com os reagentes fixados na tira-teste sendo o resultado da reação depois analisado. As primeiras tiras-teste foram desenvolvidas em 1965, separadamente pelas empresas Ames e Boehringer Mannheim (BM), as quais usavam a enzima glucose oxidase encapsulada numa membrana semipermeável como catalisador para bloquear a passagem dos glóbulos vermelhos [6]. A tonalidade da cor do produto da reação era proporcional à concentração de g'lucose. A gota de sangue aplicada na tira era removida com algodão após 1 minuto e a cor comparada com uma 
escala disponível no frasco após 1 minuto de espera para se obter um resultado semiquantitativo. Um exemplo destas tiras é mostrado na Figura 2.

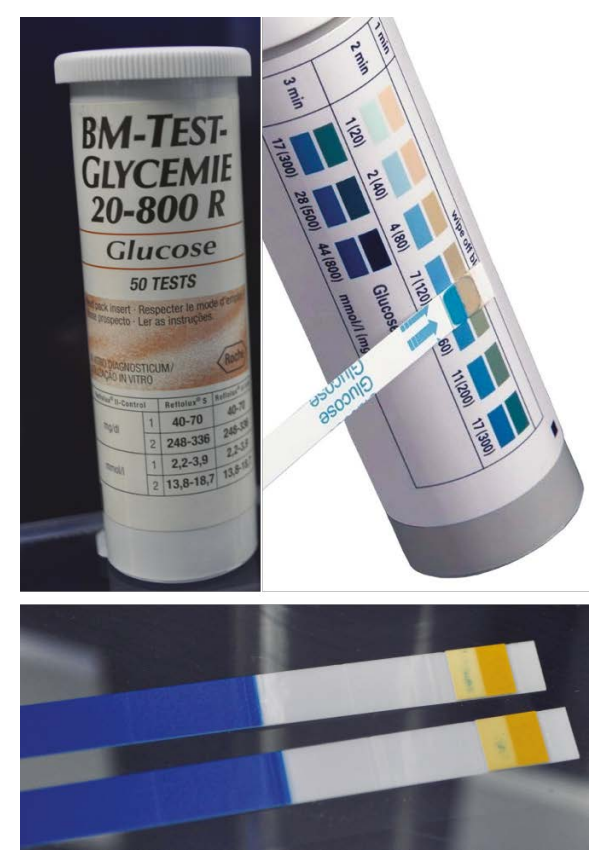

Figura 2 - BM teste (Museu da Associação Protectora dos Diabéticos de Portugal).

O passo seguinte foi o desenvolvimento, em 1975, de medidores de g'licemia que já permitiam uma determinação quantitativa e que constituíram um incremento significativo na gestão da diabetes [6]. Os primeiros modelos, com cerca de 1,2 kg, baseavam-se na análise espectral das tiras com a determinação da intensidade da radiação refletida, sendo necessário o uso de uma tira de calibração antes das medições. Os primeiros medidores tinham diversas fragilidades, tais como necessidade de uma gota de sangue grande, dependerem de calibrações e fornecerem resultados com uma margem de erro, por vezes, significativa.

A partir de 1980 ocorreu um rápido desenvolvimento dos medidores, tornando-os mais fáceis de usar, mais portáteis, mais apelativos e com memória interna. As tiras-teste também tiveram modificações relevantes para diminuir a quantidade de sangue necessária, deixar de ser necessário limpar a gota de sangue e o uso de outras metodologias. Em 1988 foram introduzidos no mercado medidores e tiras-teste baseados em processos eletroquímicos com pré-calibração, simples de manusear, mais fiáveis e mais rigorosos [7]. Desde então, o mercado evoluiu para o uso generalizado dos processos eletroquímicos com o recurso a tiras-teste com enchimento por capilaridade.

\section{Métodos usados atualmente na determinação da glicemia}

Os métodos atuais, exemplos ilustrados na Figura 3, fornecem resultados em cerca de 5 a 15 segundos, precisam apenas de um volume de 0,3 $\mathrm{LL}$ de gota de sangue e envolvem os seguintes passos:

1) Inserir a tira-teste no medidor que se liga automaticamente;

2) Obter uma gota de sangue por punção capilar;

3) Aproximar a gota de sangue da tira-teste para o mesmo ser inserido na tira por capilaridade;

4) 0 resultado da glicemia é mostrado no visor do medidor ou em outro recetor tal como uma aplicação no telemóvel.

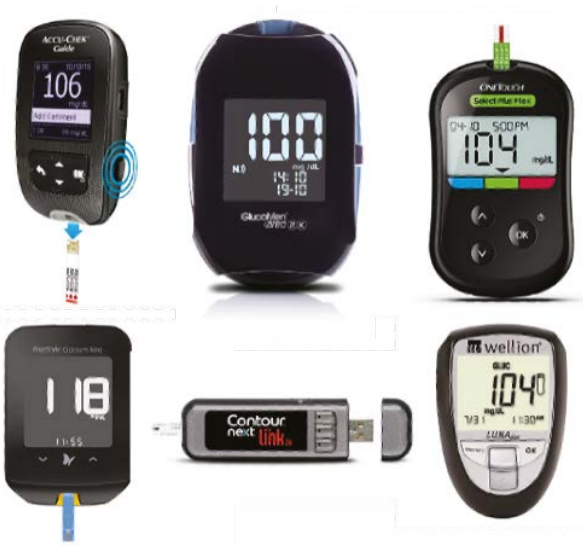

Figura 3 - Exemplos de medidores de glicemia atuais. Adaptada de imagens públicas em sítios da internet.

São usados métodos eletroquímicos para a quantificação da glicemia com a utilização de enzimas específicas para a glucose, como por exemplo a glucose oxidase ou g'lucose desidrogenase (GDH), a qual é oxidada a gluconolactona, sendo os eletrões transferidos para um mediador. Este mediador é, regra geral, uma molécula pequena que consegue rapidamente aceitar e doar eletrões permutando assim entre as suas formas oxidada e reduzida. São usados, por exemplo, derivados de ferroceno, no caso de haver um eletrão a ser permutado, ou quinonas que permutam dois eletrões.

O mediador permuta de seguida os eletrões com um elétrodo presente na tira-teste produzindo uma corrente elétrica proporcional à concentração de glucose. Os mediadores devem ter um potencial redox baixo para permitir que o elétrodo de medida funcione a baixos potenciais reduzindo as interferências e aumentado a exatidão do resultado. As reações ocorrem na superfície das camadas da tira-teste onde os processos de difusão desempenham um papel 
determinante para que o transporte das espécies seja efetuado de forma eficaz e rápida.

Nos dispositivos que usam processos colorimétricos, este fluxo de eletrões é captado por uma molécula indicadora que vai desenvolver uma cor proporcional à concentração de glucose. A Figura 4 apresenta um esquema genérico destes processos.

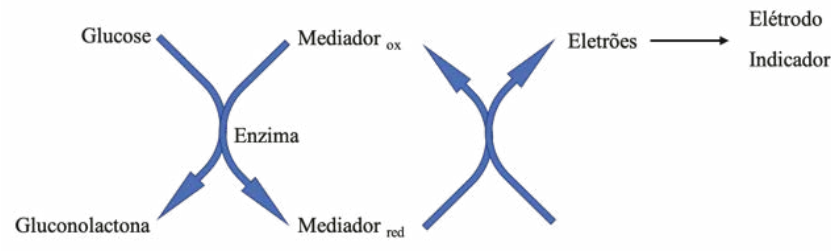

Figura 4 - Esquema genérico dos processos eletroquímicos usados nas tiras teste. Adaptada da referência [7]

Neste processo podem também ser usadas coenzimas, tais como dinucleotídeo de flavina adenina (FAD), pirroloquinolina quinona (PQQ) ou nicotinamida adenina dinucleótido (NAD), que participam nos passos intermédios do processo, tal como no exemplo mostrado na Figura 5.

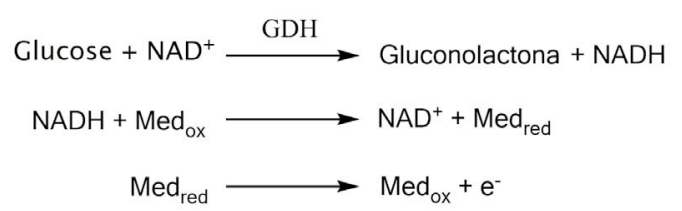

Figura 5 - Reações usadas para determinação da glicemia. Adaptada das referências $[7,9]$.

As tiras-teste contêm os reagentes e elétrodos na forma de camadas constituídas por materiais porosos como a poliamida, poliolefina, polissulfona ou celulose. Há vários processos industriais para produzir tiras-teste, tais como a impressão de tela (screen printing), revestimento de lâmina (blade coating) ou a ablação a laser, sendo o mais simples a impregnação de uma membrana com uma solução tampão contendo os reagentes.

0 desenho das tiras-teste depende do fabricante, em particular no que diz respeito ao número de elétrodos e à forma de deposição dos mesmos na tira. 0 método mais genérico usa dois elétrodos, o elétrodo de trabalho de carbono, ouro ou platina (o qual tem um revestimento com uma mistura da enzima e do mediador) e o elétrodo de referência de $\mathrm{Ag} / \mathrm{AgCl}$. As tiras-teste podem ser vistas como uma célula eletroquímica em miniatura, tal como ilustrado na Figura 6 , sendo compostas por suportes de material poroso onde os elétrodos e reagentes são ancorados ou incluídos em revestimentos. Possuem também um espaçador que separa aqueles suportes e por onde o sangue vai entrar na tira-teste. Para além dos reagentes já referidos, são também usados conservantes, para aumentar o prazo de validade, e surfactantes, para ajudar o sangue a penetrar na tira-teste.

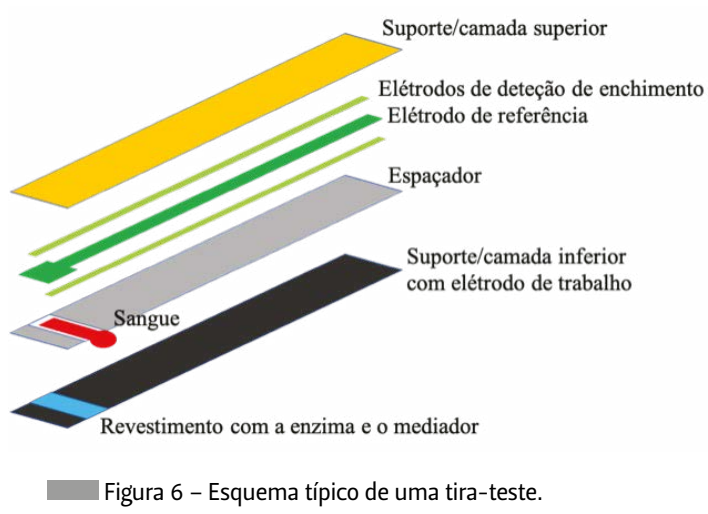

A reação tem início quando o sangue entra na tira e há uma subida da corrente elétrica, a qual atinge um máximo que decai à medida que a reação prossegue, produzindo assim uma curva de corrente em função do tempo. 0 método de análise do sinal obtido depende do desenho da tira-teste, podendo ser amperométrico ou coulométrico, onde a corrente instantânea a um tempo específico ou a carga integrada na área da curva, respetivamente, é proporcional à concentração de glucose. 0 autor e outros investigadores consideram os métodos amperométricos mais sensíveis, sendo também os mais usados.

Alguns sistemas funcionam sem o uso de mediadores, sendo a transferência de eletrões efetuada pelos elétrodos através de complexos capazes de transferir carga, tais como elétrodos de sais orgânicos condutores. O sal condutor é feito crescer num filme eletrocondutor, como por exemplo nanofitas de grafeno, sendo a enzima oxidada diretamente na superfície do cristal, o que constitui as tiras-teste [10].

Recentemente, foi publicada uma revisão bibliográfica sobre os artigos publicados entre 2016 e 2020 sobre os métodos em desenvolvimento com potencial para a determinação da g'licemia [8]. Estes métodos envolvem o uso de nanomateriais, polímeros condutores, nanotubos de carbono, grafeno, redes metal-orgânicas (MOFs) e polímeros de impressão molecular (MIPs). Estes materiais não se encontram ainda incorporados em produtos comerciais, nomeadamente devido à sua toxicidade, disponibilidade e custo acrescido, entre outros. 
Atualmente, os medidores de g'licemia têm que obedecer à norma ISO 15197, publicada em 2003 e revista em 2013, que estabelece os critérios de desempenho aceitáveis para estes dispositivos médicos [11]. Esta norma usa como critério de desempenho adequado a obtenção de, pelo menos, 95\% dos resultados no intervalo $\pm 15 \mathrm{mg} / \mathrm{dL}$, tendo como padrão a determinação laboratorial, ou dentro do limite $\pm 15 \%$ do valor padrão para valores de g'licemia maiores ou iguais a $100 \mathrm{mg} / \mathrm{dL}$.

Os métodos anteriores fornecem o valor pontual da glicemia associado ao instante em que o teste é realizado. A glicemia pode também ser determinada de uma forma contínua no líquido intersticial por diversos sistemas existentes no mercado. Estes medidores contínuos de glicemia, ilustrados na Figura 7, funcionam pela colocação de um sensor no tecido subcutâneo, normalmente no braço ou abdómen, os quais possuem uma membrana que permite a passagem da glucose até ao compartimento onde está armazenada a enzima g'icose oxidase. Após a reação ocorrer é originado um sinal elétrico diretamente proporcional à concentração de glucose. Esse sinal é enviado pelo transmissor (que está ligado ao sensor) para o recetor que o transforma no valor da glicemia permitindo a leitura no visor. Este processo é feito de forma contínua sendo a glicemia determinada a cada 5 minutos. 0 recetor, fornecido pela empresa, ou uma aplicação no telemóvel mostra a glicemia do momento e também um gráfico com a evolução ao longo do tempo. A comunicação do valor da glicemia pode também ser feita para uma bomba infusora de insulina. Consoante a marca, estes sensores precisam, ou não, de calibrações diárias com valores de glicemia capilar e podem ser usados entre 7 a 14 dias, sendo o transmissor recarregável e reutilizável. 0 valor da g'licemia no líquido intersticial tem um hiato temporal com a glicemia capilar de 5 a 15 minutos, motivado pela difusão da glucose do sangue para o líquido intersticial. Esta diferença é de particular relevância quando existe uma variação brusca de glicemia $[12,13]$.

\footnotetext{
Figura 7 - Medidores contínuos de glicemia. Adaptada de imagens públicas em sítios da internet.
}
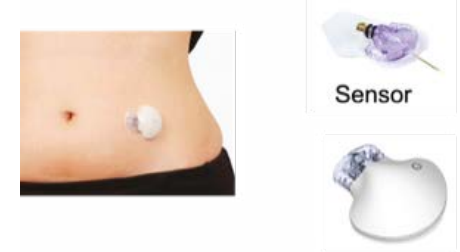

Sensor + transmisso

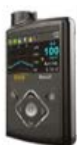

Bomba infusora de insulina
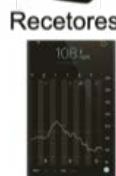

Os sistemas contínuos têm algumas vantagens importantes pois mostram a evolução da g'licemia ao longo do tempo, permitindo um melhor controlo da diabetes e um melhor ajuste da terapêutica. Estes sistemas têm um conjunto de alarmes que são acionados quando a g'licemia tem variações bruscas e quando está acima ou abaixo de determinado valor inserido pelo utilizador. Esta é uma vantagem fundamental em pessoas com diabetes sem sensibilidade para hipoglicemias e que sem esta monitorização podem entrar em coma pelo seu não tratamento adequado e atempado. Um exemplo da curva obtida em comparação com os valores de ǵlicemia discretos é mostrado na Figura 8. Para saber mais sobre os sistemas disponíveis em Portugal pode consultar os sítios de internet de algumas empresas comercializadoras [14-16].

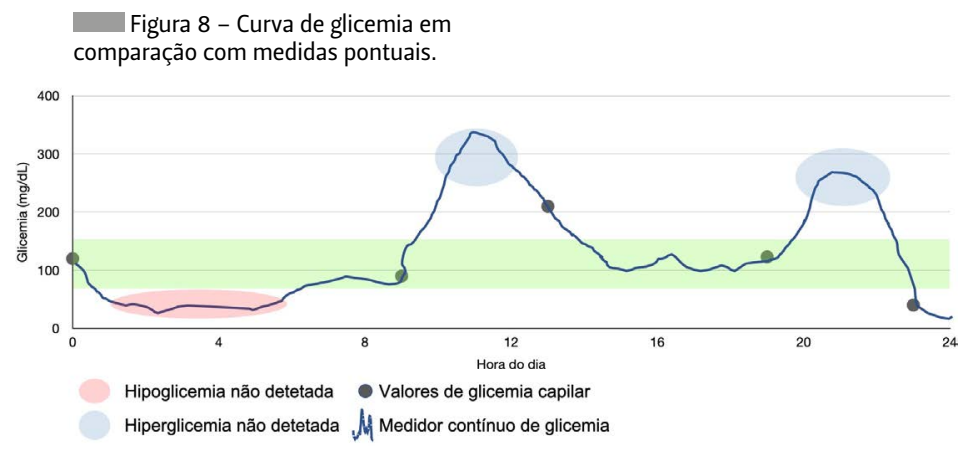

Surgiu no mercado nos últimos anos um medidor com um sistema flash para medição da glicemia. Este sistema é composto por um sensor introduzido no tecido subcutâneo, o qual tem um transmissor acoplado. Os dados são transmitidos por NFC (protocolo de comunicação entre aparelhos por aproximação, Near Field Communication) para um leitor, ou um telemóvel com a aplicação, quando estes são aproximados do transmissor. 0 sistema mede a glicemia a cada minuto e guarda os dados das últimas 8 horas. Quando ocorre a transmissão dos dados, o leitor mostra a glicemia no momento, a curva das g'licemias das últimas 8 horas e uma seta de tendência com indicação da evolução mais recente. Este sistema mede a glicemia no tecido intersticial e usa a glucose oxidase e mediadores de ósmio inseridos numa matriz polimérica constituindo uma rede complexa de terminais sensíveis à glucose, não precisando de calibrações diárias (Figura 9). Cada sensor tem uma duração de 14 dias. Para saber mais pode consultar o sítio de internet da empresa Abbott [17].

Foi desenvolvido um sistema de medição contínua implantado no braço e com a duração de uso até seis meses na versão mais recente. Este medidor mede a glucose no líquido intersticial através da reação com o oxigénio molecular, catalisada pela g'lucose oxidase 
imobilizada em grafite, para formar gluconolactona e peróxido de hidrogénio. 0 aumento da concentração de peróxido de hidrogénio e a diminuição da concentração de oxigénio é proporcional à concentração de glucose [10]. Para saber mais pode consultar o sítio de internet da empresa Eversense [18].

Em desenvolvimento estão duas tecnologias, já publicitadas nos meios de comunicação e alguns artigos, designadamente lentes de contacto com sensores e tatuagens [19]. No primeiro caso, a ser desenvolvido pela Google e pela Novartis, a lente tem incorporado um sensor que usa as lágrimas como meio de análise e um transmissor [20]. No caso das tatuagens, estão a ser desenvolvidos pigmentos sensíveis à concentração de glucose a serem incorporados nas tintas usadas nas tatuagens. Estes pigmentos mudam de cor consoante a g'icemia e podem ser usados como uma medida semiquantitativa deste valor. Estão também em estudo, ainda laboratorial na esmagadora maioria dos casos, métodos não invasivos para determinação da ǵlicemia que tentam explorar a resposta da glucose a diferentes tipos de radiação, tais como ultrassons, radiofrequências e infravermelho e técnicas espectroscópicas aliadas a inteligência artificial [21].
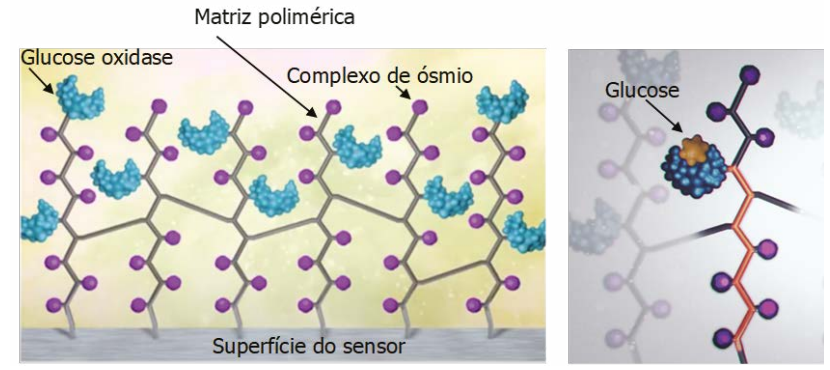

Figura 9 - Sistema em rede usado pelo Freestyle Libre ${ }^{\circledR}$.

\section{Agradecimentos}

Agradeço à Associação Protectora dos Diabéticos de Portugal a visita ao museu e a disponibilidade para a obtenção das fotografias e sua utilização neste artigo.

\section{Referências}

[1] "Viver com a diabetes, 3. ${ }^{a}$ Edição", Associação Protetora dos Diabéticos de Portugal, Lidel-Edições Técnicas Lda, Lisboa, 2012.

[2] Norma da Direção Geral da Saúde. Disponível em: dgss.pt/programa-nacionalpara-a-diabetes/circulares-normas-e-orientacoes/norma-da-direccao-geralda-saude-n-0022011-de-14012011-pdf.aspx (acedido em 18/06/2020).

[3] "IDF Diabetes Atlas, 9 $9^{\text {th }}$ Ed." International Diabetes Federation, Bruxelas, 2019.

[4] "Diabetes factos e números - ano 2015", Observatório Nacional da Diabetes, Lisboa, 2016. Disponível em: apdp.pt/publicacoes/relatorio-anual-doobservatorio-nacional-da-diabetes-2016.

[5] The Diabetes Control and Complications Trial Research Group, N. Engl. J. Med. 1993, 329, 977-986. DOI: 10.1056/NEJM199309303291401.

[6] S. F. Clarke, J. R. Foster, Br. J. Biomed. Sci. 2012, 69, 83-93. DOI: 10.1080/09674845.2012.12002443.

[7] J. Hones, P. Muller, N. Surridge, Diabetes Techno. Ther. 2008, 10, S10-S26. DOI: 10.1089/dia.2008.0005.

[8] E. Sehit, Z. Altintas, Biosens. Bioelectron. 2020, 159, 112165. DOI: 10.1016/j. bios.2020.112165

[9] J. Wang, Chem. Rev. 2008, 108, 814-825. DOI: 10.1021/cr068123a.

[10] C. Sabu, T. K. Henna, V. R. Raphey, K. P. Nivitha, K. Pramod, Biosens. Bioelectron. 2019, 141, 111201. DOI: 10.1016/j.bios.2019.03.034.

[11] International Organization for Standardization. ISO 15197: 2013(E): In vitro diagnostic test systems-requirements for blood-glucose monitoring systems for self-testing in managing diabetes mellitus. Geneva, Switzerland: International Organization for Standardization, 2013

[12] P. Apablaza, N. Soto, R. Román, E. Codner, Rev. Med. Clin. Condes 2016, 27, 213-226. DOl: 10.1016/j.rmclc.2016.04.011.

[13] J. J. Kannampilly, Continous Glucose Monitoring System, 198-200 in A. Muruganathan (ed.) "Medicine Update", Jaypee Brothers Medical Publishers, 2013.

[14] Dexcom Continuous Glucose Monitoring: dexcom.com (acedido em 29/03/2021).

[15] Medtronic: medtronic-diabetes.com.pt (acedido em 29/03/2021).
[16] A. Menarini diagnstics: menarinidiagnostics.com/en-us/Home/Diabetescare-products/Glucomen-day-CGM/Features (acedido em 29/03/2021).

[17] Freestyle: provider.myfreestyle.com/freestyle-libre-2-accuracy.html (acedido em 29/03/2021).

[18] Eversense: eversensediabetes.com/sensor (acedido em 29/03/2021).

[19] D. Meetoo, L. Wong, B. Ochieng, Br. J. Nurs. 2019, 28, 110-115. DOl: 10.12968/ bjon.2019.28.2.110.

[20] M. Senior, Nat. Biotechnol. 2014, 32, 856. DOI: 10.1038/nbt0914-856.

[21] W. V. Gonzales, A. T. Mobashsher, A. Abbosh, Sensors 2019, 19, 800. DOl: 10.3390/s19040800.

$>$

\section{João Valente Nabais}

João V. Nabais é Professor Auxiliar com Agregação da Universidade de Évora. Desenvolve o seu trabalho de investigação no Comprehensive Health Research Center. Tem desenvolvido atividades letivas e de promoção da ciência na Química Forense. É Vice-Presidente da Federação Internacional da Diabetes desde 2019. jvn@uevora.pt ORCID.org/0000-0001-9572-6346 